\title{
Diseño e implementación de un controlador no-lineal para un brazo robótico con articulaciones flexibles y enlaces rígidos
}

Design and implementation of a nonlinear controller for a robotic arm with flexible joints and rigid links

Juan Jose Padilla-Mora ${ }^{1}$

Padilla-Mora, J. J. Diseño e implementación de un controlador no-lineal para un brazo robótico con articulaciones flexibles y enlaces rígidos. Tecnología en marcha. Edición especial Movilidad Estudiantil 7. Abril, 2020.

Pág.160-168.

doi https://doi.org/10.18845/tm.v33i6.5312 


\section{Palabras clave}

Sistema mecánico; control no-lineal; sistema Hamiltoniano; Robótica; Sistema singularmente perturbado; sistema rápido-lento.

\section{Resumen}

Los sistemas robóticos se están volviendo cada vez más complejos y las teorías de la ley de control tradicional pierden robustez, lo que aumenta la dificultad con la que se puede controlar el robot para interactuar con el entorno que lo rodea. El objetivo de este trabajo de investigación es el estudio de sistemas no lineales complejos con la particularidad de tener articulaciones flexibles y enlaces rígidos. Dicha flexibilidad provoca un comportamiento interesante en los sistemas robóticos porque duplica el número de variables involucradas en la tarea de control.

Se han llevado a cabo varios estudios en la investigación de robots flexibles, sin embargo, la mayoría de ellos utilizan el marco clásico de Euler-Lagrange para describir los sistemas mecánicos. Este trabajo se ha centrado en la implementación de controladores no lineales dentro del marco portuario-hamiltoniano, y en la teoría de sistemas de perturbación singular a escala múltiple.

En este sentido, la descripción matemática de dos leyes de control diferentes propuestas por [1] y [2] se presentan y adaptan a la planta física del brazo robótico Quanser de dos grados de libertad.

Además, las ecuaciones de los controladores port-hamiltonianos propuestos se han implementado en una simulación para probar la validez de las leyes de control para la configuración rígida y flexible del robot. Finalmente, los controladores se han implementado en la planta física del brazo robótico para validar experimentalmente la teoría matemática de control propuesta.

La implementación experimental de los controladores puerto-hamiltonianos propuestos mostró una mejora en el control del error de posición para la configuración rígida y flexible en comparación con un controlador de referencia propuesto por el fabricante del brazo robótico, con una tasa de error para el valor RMS de la señal inferior al 1,2\% del valor RMS de la trayectoria deseada. Otros estudios y pruebas experimentales deben estar dirigidos a la implementación de controladores porto-hamiltonianos para lograr una tasa de error aún más baja.

\section{Keywords}

Mechanic system; non-linear control; Hamiltonian system; Robotics; Uniquely disturbed system; fast-slow system.

\section{Abstract}

Robotic systems are becoming more and more complex and the traditional control law theories lose robustness, increasing the difficulty with which the robot can be controlled to interact with the environment around it. The objective of this research work is the study of complex nonlinear systems with the particularity of having flexible joints and rigid links. Such flexibility causes an interesting behavior in the robotic systems because duplicates the number of variables involved in the control task.

Several studies have been carried out in the research of flexible robots, however most of them use the classical Euler-Lagrange framework to describe the mechanical systems. This work 
has been focused on the implementation of nonlinear controllers within the port-Hamiltonian framework, and the singular perturbation multi-scale systems theory.

In this sense, the mathematical description of two different control laws proposed by [1] and [2] are presented and adapted to the physical plant of the two degrees of freedom Quanser robotic arm.

Moreover, the equations of the proposed port-Hamiltonian controllers have been implemented into a simulation to test the validity of the control laws for the rigid and the flexible configuration of the robot. Finally, the controllers have been implemented into the physical plant of the robotic arm to validate experimentally the proposed mathematical control theory.

The experimental implementation of the proposed port-Hamiltonian controllers showed an improvement in the control of the position error for the rigid and the flexible configuration in comparison with a benchmark controller proposed by the manufacturer of the robotic arm, with an error rate for the RMS value of the signal lower than $1.2 \%$ of the RMS value of the desired trajectory. Further studies and experimental tests should be aimed to the implementation of portHamiltonian controllers to achieve an even lower error rate.

\section{Estado del arte}

La mayoría de los estudios en el campo de la robótica flexible se basan en el modelo clásico de la ecuación de Euler-Lagrange, como se muestra en [3], [4] y [5]. Este método presenta problemas porque las cantidades involucradas no tienen interpretación física, lo que aumenta la dificultad en la implementación de controladores.

Por otro lado, cuando se dimensionan, los robots flexibles presentan la dificultad que sus modelos son al menos dos veces más complejos que los sistemas rígidos, lo que complica su estudio. La teoría de los sistemas singularmente perturbados fue desarrollada para abordar el análisis de estos sistemas, proporcionando técnicas para reducir la matemática en el modelo de robots flexibles en [6], [7] y [8]. Sin embargo, esta teoría no es suficiente para establecer un modelo matemático decisivo para un sistema flexible.

Dada esta dificultad, se plantea un nuevo enfoque basado en dar forma a la energía del sistema. Este nuevo paradigma se llama: sistemas hamiltonianos controlados por puertos. En [9] se menciona que no es hasta hace poco que los investigadores lograron integrar sistemas singularmente perturbados con sistemas Hamiltonianos controlados por puertos. Dicha integración facilitaría en gran medida el análisis matemático de modelos para robots flexibles.

\section{Configuración experimental}

La configuración experimental consta de dos motores de CD, cada uno con un enlace serie de dos barras. El enlace primario está acoplado al primer accionamiento por medio de una junta flexible y lleva al final el segundo accionamiento armónico, al que está unido el segundo enlace rígido. La junta flexible puede atascarse para asegurar una junta rígida.

El manipulador tiene enlaces con longitudes $l_{i}$, ángulos $\theta_{i}$, masa $m_{i}$, distancia al centro de la masa $r_{i}$ y momento de inercia $I_{i}$ con $i=1,2$. Mientras el sistema funciona en el plano horizontal, las fuerzas gravitacionales se desprecian. Finalmente, las articulaciones se han definido como $J_{1}$ y $J_{2}$, la posición de los enlaces como $q_{1}$ y la posición de motor como $q_{m 1}$. La figura 1 muestra el diagrama del modelo y la figura 2 muestra la planta física. 


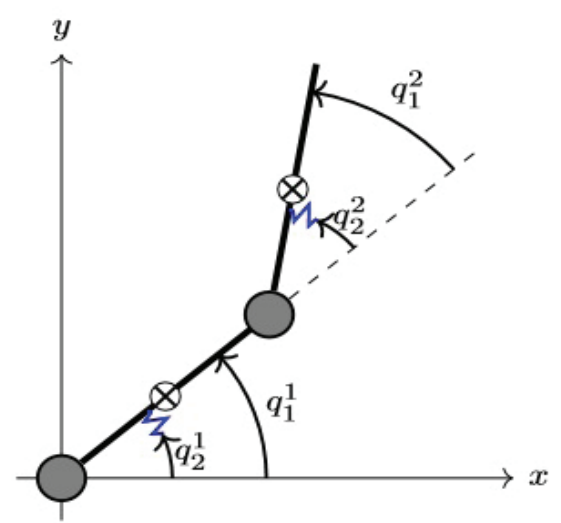

Figura 1. Esquematico de 2DoF Manipulador Quanser [10].

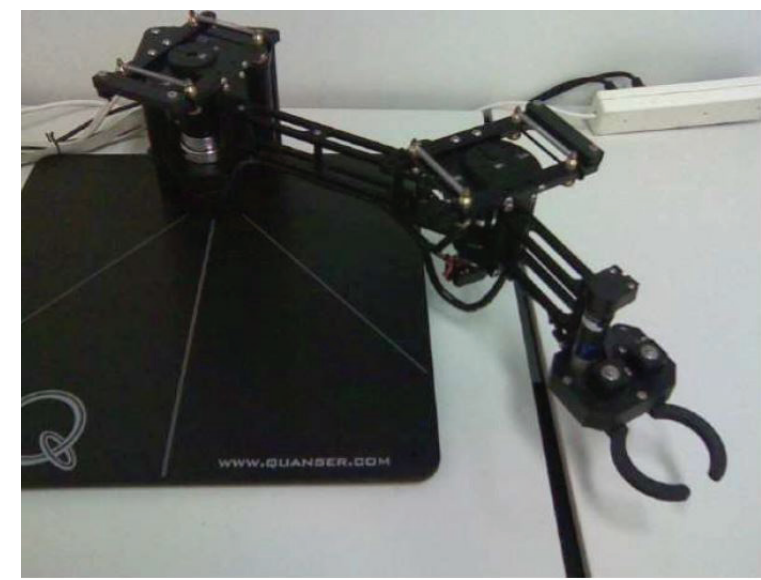

Figura 2. Planta física del 2DoF Manipulador Quanser [10].

\section{Modelo matemático Hamiltoniano por puertos}

El sistema puede describirse en la estructura de $\mathrm{PH}$ en términos de sus coordenadas y sus momentos. Pertenece al tipo de sistemas expandidos de la mecánica clásica detallados en [11], por lo tanto, pueden describirse como:

$$
\left[\begin{array}{c}
\dot{q} \\
\dot{p}
\end{array}\right]=\left[\begin{array}{cc}
0 & I \\
-I & -D(q, p)
\end{array}\right]\left[\begin{array}{l}
\frac{\partial H(q, p)}{\partial q} \\
\frac{\partial H(q, p)}{\partial p}
\end{array}\right]+\left[\frac{0}{B(q)}\right] u
$$

Para la configuración flexible, la expresión matemática para el hamiltoniano debe expandirse para incluir no solo la energía cinética del sistema, sino también la energía potencial almacenada en los resortes, como en [1]. El sistema expandido tiene una estructura lenta-rápida descrita en términos de variables hamiltonianas, por lo tanto, la entrada de control u se definirá, así como una estructura de $\mathrm{PH}$ con un error de sistema adecuado. 


$$
\left[\begin{array}{c}
\dot{q} \\
\dot{z} \\
\dot{p} \\
\dot{\gamma}
\end{array}\right]=\left[\begin{array}{cc}
0_{4 x 4} & I_{4 x 4} \\
-I_{4 x 4} & -D_{4 x 4}(q, p)
\end{array}\right]\left[\begin{array}{l}
\frac{\partial H(q, z, p, \gamma)}{\partial q} \\
\frac{\partial H(q, z, p, \gamma)}{\partial z} \\
\frac{\partial H(q, z, p, \gamma)}{\partial p} \\
\frac{\partial H(q, z, p, \gamma)}{\partial \gamma}
\end{array}\right]+\left[\begin{array}{c}
0 \\
0 \\
0 \\
B(q)
\end{array}\right] u
$$

\section{Controlador multi-escala Reyes-Baez}

Esta sección sigue la teoría matemática para el diseño de la ley de control de un controlador multi-escala que utiliza el método de control basado en contracción para el sistema lento y el controlador propuesto por [12] como el controlador rápido.

Como se afirma en [2], el controlador propuesto conserva la estructura de un sistema hamiltoniano que combina las técnicas de estabilidad transitoria exponencial descrita en [13] y el enfoque de colectores deslizantes descrito en [14]. La idea es crear una ley de control de contracción donde un plano invariante se vuelve atractivo y otra ley de control que asegura que en ese plano invariante, la trayectoria sigue el movimiento deseado.

Como en [12], se proponen nuevas coordenadas para el sistema como $\left(q_{1}, \epsilon_{z}\right)=$

$\left(q_{1}, q_{1}-q_{2}\right)$ para lograr un modelo de perturbación singular lento-rápido. Además, las coordenadas generalizadas se han definido como $q_{\epsilon}=\left(q_{1}, z\right)$. Las dinámicas de control vienen dadas por:

$$
\begin{gathered}
\dot{\tilde{q}}=M^{-1}(\sigma-\wedge \tilde{q}) \\
\dot{\sigma}=-\left[\frac{\partial H}{\partial q}(x)+D(q) \frac{\partial H}{\partial p}(x)-G(q) U_{s}+\dot{p}_{r}\right]
\end{gathered}
$$

Y la ley de control viene dada por:

$$
\begin{gathered}
U_{S R}=u_{e q}-u_{a t} \\
u_{e q}=\dot{p}_{r}+\frac{\partial H}{\partial q}\left(q, p_{r}\right)+D(q) \frac{\partial H}{\partial p}\left(q, p_{r}\right) \\
u_{a t}=-K_{d} \frac{\partial H}{\partial p}(q, \sigma)-M^{-1}(q) \wedge \tilde{q}+\frac{\partial}{\partial q}\left(p_{r}{ }^{T} M^{-1}(q) \sigma\right) \\
U_{f s}=K_{v}\left(\dot{q}-\dot{q}_{m}\right)
\end{gathered}
$$




\section{Controlador multi-escala Jardón-Kojakhmetov}

En esta sección se utiliza el controlador propuesto por [15] como controlador lento, y además propone una ley de control rápida con la misma estructura, así como el sistema multi-escala que une los dos subsistemas. Para resolver el problema de control, el autor deriva un modelo de $\mathrm{PH}$ utilizando una transformación de coordenadas similar al controlador Reyes-Baez.

La idea es establecer una ley de control para el seguimiento de trayectoria utilizando solo la medición de posiciones. Para esta tarea, y como se indica en [15], una dinámica de retroalimentación y una matriz de Coriolis se aplican usando las velocidades de referencia en lugar de las velocidades reales del sistema.

Como en [12], se proponen nuevas coordenadas para el sistema como $\left(q_{1}, \epsilon_{z}\right)=$

$\left(q_{1}, q_{1}-q_{2}\right)$. Asimismo, las nuevas coordenadas generalizadas se han definido como $q_{\epsilon}=\left(q_{1}, z\right)$. Las dinámicas de control vienen dadas por:

$$
\left[\begin{array}{c}
\dot{\tilde{q}}_{\epsilon} \\
\tilde{p}_{\epsilon}
\end{array}\right]=\left[\begin{array}{cc}
0_{n x n} & -\widetilde{T}_{\epsilon}\left(\tilde{q}_{\epsilon}\right)^{-\top} \\
-\widetilde{T}_{\epsilon}\left(\tilde{q}_{\epsilon}\right)^{-1} & \tilde{J}_{\epsilon}\left(\widetilde{q}_{\epsilon}\right)-\widetilde{D}_{\epsilon}\left(\tilde{q}_{\epsilon}\right)
\end{array}\right]\left[\begin{array}{c}
\frac{\partial \widetilde{H}\left(\tilde{q}_{\epsilon}, \tilde{p}_{\epsilon}\right)}{\partial \tilde{q}_{\epsilon}} \\
\frac{\partial \widetilde{H}\left(\tilde{q}_{\epsilon}, \tilde{p}_{\epsilon}\right)}{\partial \tilde{p}_{\epsilon}}
\end{array}\right]+\left[\frac{0_{n x n}}{G\left(\tilde{q}_{\epsilon}, \tilde{p}_{\epsilon}\right)}\right] \tilde{u}
$$

Y la ley de control viene dada por:

$$
\tilde{u}=M(q) \ddot{q}_{d}+\frac{\partial(M(q) \dot{q})}{\partial q} \dot{q}-\frac{1}{2} \frac{\partial \dot{q}^{\top} M(q) \dot{q}}{\partial q}-K_{p} \tilde{q}-K_{c} v
$$

\section{Experimento de pulso}

Para esta prueba, ambas articulaciones fueron dirigidas a una posición fija de $30^{\circ}$. Para el controlador multi-escala Reyes-Baez los parámetros utilizados fueron $K d=\operatorname{diag}(-5,-1), \lambda=$ $\operatorname{diag}(50,5), K v=\operatorname{diag}(-14,-16)$ and $\epsilon=0.3$. Para el controlador multi-escala Jardon-Kojakhmetov los parámetros utilizados fueron $K d=\operatorname{diag}(3,3), K c=\operatorname{diag}(100,100), K p=\operatorname{diag}(300,200), L d=$ $\operatorname{diag}(3,3), L c=\operatorname{diag}(300,300)$ and $L p=\operatorname{diag}(200,100)$ and $\epsilon=0.03$. La trayectoria deseada se puede ver en la figura 3.

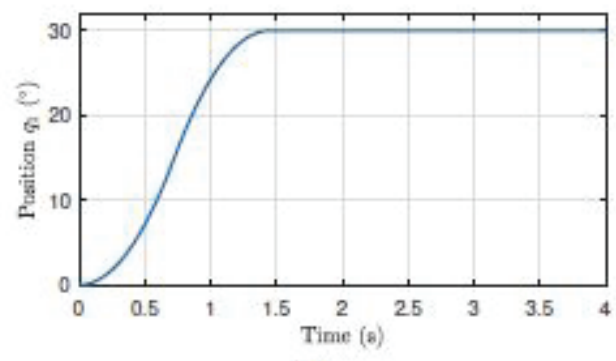

(a)

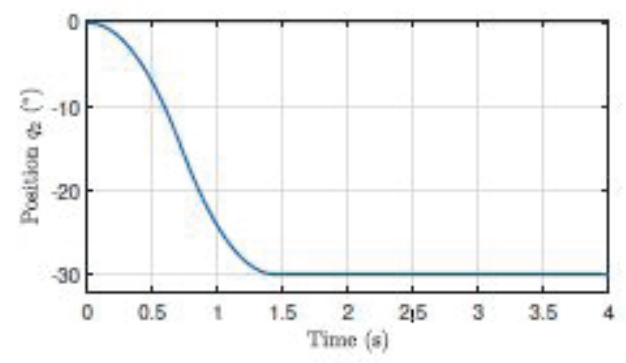

(b)

Figura 3. Experimento de pulso, trayectoria de posición de la articulación 1 (a) y trayectoria de posición de la articulación 2 (b). 
Los gráficos correspondientes a los resultados experimentales (ver figuras 4 y 5) se obtuvieron mediante la medición de la posición de los servomotores que controlaban el movimiento del brazo robótico y la recolección de datos en Matlab. La prueba de pulso se realizó con el objetivo de analizar la precisión en el estado estacionario y la velocidad con la que el error de posición y el error flexible convergen a cero. Como se puede apreciar en la figura 4 y especialmente en la figura 5, si bien el controlador multi-escala Reyes-Báez converge tras 2.5s no puede amortiguar las oscilaciones en el estado transitorio, a diferencia de; controlador multi-escala JardónKojakhmetov que parece tener la mejor respuesta. Esta mejora en la convergencia del error se debe al mejor control del error flexible para debido a que tiene la misma estructura que un sistema rígido; el controlador rápido tiene una estructura diseñada siguiendo el mismo modelo que el controlador lento, a diferencia del controlador rápido Spong utilizado en el controlador Reyez-Baez que tiene solo una ganancia proporcional.

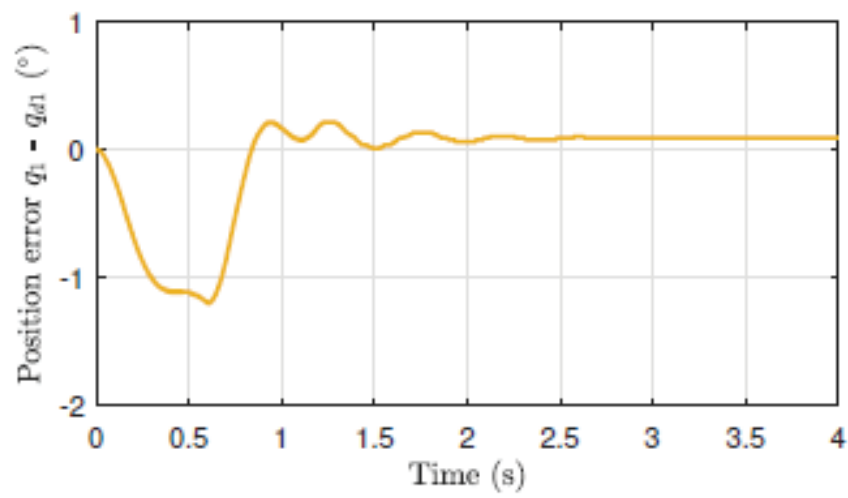

(a)

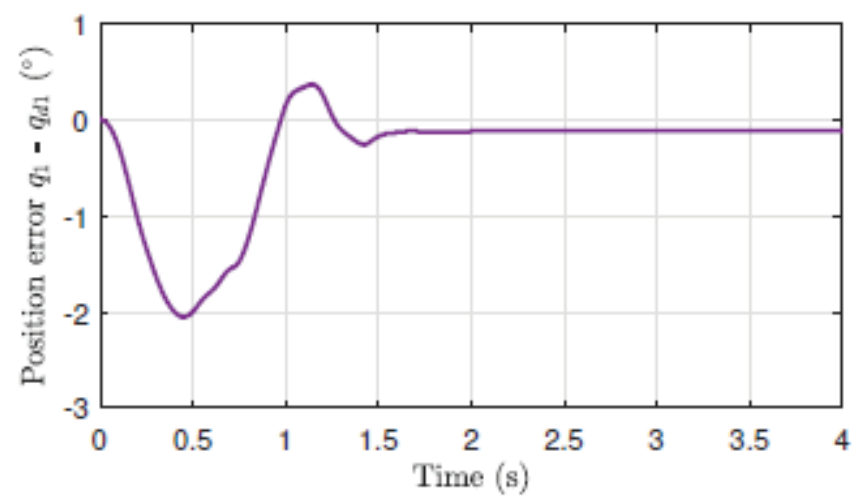

(b)

Figura 4. Error de posición para la articulación 2.

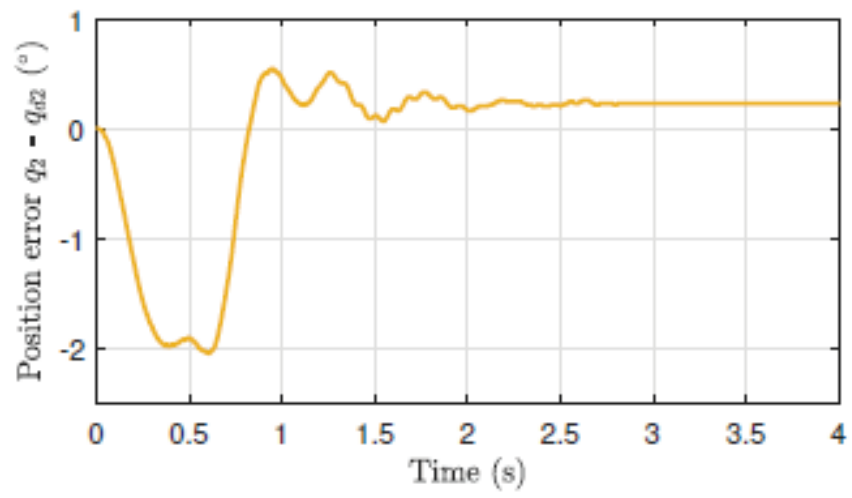

(a)

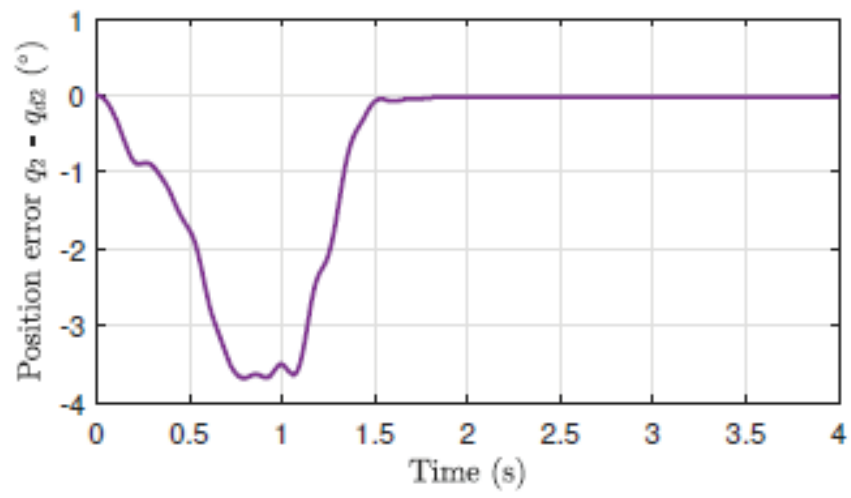

(b)

Figura 5. Error de posición para la articulación 2. 


\section{Conclusiones}

La teoría matemática para los controladores de Hamiltonianos controlados por puertos en [1], [15] y [2] se ha adaptado al brazo robótico 2DoF Quanser [10] para la configuración rígida, y se ha expandido para la configuración flexible. Además, la descripción matemática del manipulador Quanser se ha resumido para facilitar el trabajo futuro con la planta física.

Además, el modelo matemático para el robot manipulador 2DoF Quanser [10] y los controladores de $\mathrm{PH}$ propuestos se han simulado para probar el rendimiento de los controladores propuestos para la configuración rígida y flexible. Aunque en ambos casos se ha satisfecho el tiempo de asentamiento deseado, el controlador Dirksz y Scherpen [15] presentó el mejor rendimiento.

La principal contribución de esta investigación consiste en las pruebas experimentales realizadas después de la implementación física de los controladores de PH en el robot manipulador 2DoF Quanser. Hasta la fecha de publicación de esta investigación, no hay un solo experimento similar realizado con PH Controladores para un robot flexible. Para el controlador Reyes-Báez et al. [2] es la primera vez que los resultados se logran después de la implementación experimental del controlador. Para los controladores rígidos [15], los resultados experimentales logrados por esta investigación son muy superiores al trabajo previo realizado en [16] en términos de error de posición. Para la configuración flexible, ambos controladores de $\mathrm{PH}$ se han implementado físicamente por primera vez y se han cumplido los parámetros establecidos en los objetivos, con el controlador Jardon-Kojakhmetov como el controlador multiescala que muestra el mejor sistema respuesta.

\section{Referencias}

[1] Jardon-Kojakhmetov, H., Munoz-Arias, M., and Scherpen, J. M. A. (2017). Model reduction of a flexible-joint robot: a port-Hamiltonian approach.

[2] Reyes-Baez, R., van der Schaft, A., and Jayawardhana, B. (2016). Tracking Control of Fully-actuated Mechanical port-Hamiltonian Systems using Sliding Manifolds and Contraction.

[3] Canudas de Vit, C., Siciliano, B., and Bastin, G. (1996). Theory of robot control. Springer.

[4] De Luca, A. (2014). Flexible Robots. Encyclopedia of Systems and Control.

[5] Spong, M. W. (2014). Modeling and Control of Elastic Joint Robots. Journal of Dynamic Systems, Measurement, and Control.

[6] Kokotovic, P., Khalil, H., and O'Reilly, J. (1986). Singular Perturbation methods in Control: Analysis and Design.

[7] Kokotovic, P. (1984). Applications of Singular Perturbation techniques to Control problems. 26(4):501-550.

[8] Kokotovic, P., O'Malley, R., and Sannuti, P. (1976). Singular perturbation and order reduction in control theory An overview. 12(2):123-132.

[9] Van Der Schaft, A. and Maschke, B. (2003). Port-Hamiltonian systems: a theory for modeling, simulation and control of complex physical systems.

[10] Quanser (2006). 2DOF Serial Flexible Joint Robot. Reference Manual.

[11] Van Der Schaft, A. (2000). Port-controlled Hamiltonian systems: towards a theory for control and design of nonlinear physical systems. Journal of the Society of Instrument and Control Engineers of Japan (SICE), 39(2):91-98.

[12] Spong, M. W. (1990). Control of flexible joint robots: a survey. technical, University of Illinois at UrbanaChampaign.

[13] V. Andrieu, B. Jayawardhana \& L. Praly. Transverse exponential stability and applications. IEEE Transactions on Automatic Control, 2016.

[14] Ghorbel, F. and Spong, M. (2016). Integral manifolds of singularly perturbed systems with application to rigidlink flexible-joint multibody systems. International Journal of Nonlinear Mechanics. 
[15] Dirksz, D. A. and Scherpen, J. M. A. (2013). On tracking control of rigid-joint robots with only position measurements. IEEE Transactions on Control Systems Technology.

[16] Van Logtestijn, M. (2010). Stabilization and tracking control of a planar robot withonly position measurements. bachelor thesis, University of Groningen. 